\title{
Teorías analíticas de las emociones: el debate actual y sus precedentes históricos
}

\section{Analytical theories of emotions: The current debate and its historical background}

\author{
ÍNGRID VENDRELL FERRAN \\ Universidad Complutense
}

Recibido: 12-08-2008 Aprobado definitivamente: 24-11-2008

\section{RESUMEN}

Durante los últimos años, la filosofía analítica ha centrado su interés en la temática de las emociones y se han elaborado las más diversas teorías. En este artículo me propongo, por un lado, exponer las principales líneas de investigación actuales sobre las emociones y desarrollar los argumentos en favor y en contra cada una de ellas con el fin de perfilar mi propia posición; por otro lado, voy a presentar los desarrollos históricos precedentes a las teorías analíticas mostrando que la historia de la filosofía no ha relegado las emociones al olvido.

PALABRAS CLAVE

EMOCIÓN, FILOSOFÍA ANALÍTICA, TEORÍAS DEL SENTIR, TEORÍAS COGNITIVISTAS.

\begin{abstract}
Emotions are one of the topics that have caught the attention of analytical philosophy during the past years, and philosophers have developed different theories in this field. In this article, I shall present the main lines of investigation in the current debate on emotions and explain the arguments in favour and against each of them in order to build up my own position on the one hand, and, present the historical developments previous to the analytical theories, showing that the history of philosophy has always had an interest in emotions as a topic on the other hand. KEY WORDS

EMOTION, ANALYTICAL PHILOSOPHY, FEELING THEORIES, COGNITIVISTIC THEORIES
\end{abstract}




\section{LA FILOSOFÍA ANALÍTICA Y EL ESTUDIO DE LAS EMOCIONES}

DESDE HACE UNOS 40 AÑos y a raíz de la publicación del artículo «Emotions» (1957) de Erroll Bedford y del libro Anthony Kenny Action, Emotion and Will (1963) las emociones se han convertido en uno de los principales temas de interés de la filosofía contemporánea. ${ }^{1}$ Entre los numerosos autores de corte analítico que se han dedicado a su estudio destacan a parte de los ya citados: Ronald de Sousa, Robert Solomon, John Elster, Martha Nussbaum, Peter Goldie y Aaron Ben-ze'ev. En el marco de la filosofía analítica se ha hablado a menudo de un redescubrimiento de las emociones afirmando con ello que éstas habían sido relegadas al olvido por los filósofos de generaciones anteriores. Como todo «nuevo» campo de investigación, las emociones despertaron el interés de muchos autores los cuales formularon las más diversas tesis sobre lo emocional. Estas teorías resultan por su singularidad y especificidad difíciles de ubicar dentro de un mismo marco teórico haciendo casi imposible al interesado en la temática orientarse ante tan variado panorama intelectual. En este artículo me propongo un triple objetivo. Por un lado, voy a exponer las principales líneas de investigación que se esconden tras las diversas teorías actuales mostrando los argumentos y contra-argumentos a favor y en contra de ellas. Con esta labor taxonómica se pretende ofrecer una especie de mapa orientativo en el enredado panorama actual. Por otro lado, voy a ubicar las teorías de las emociones actuales en su contexto histórico, mostrando los paralelos y disimilitudes con las teorías de las emociones de los siglos inmediatamente precedentes al nuestro. Me centraré principalmente en la tradición anglosajona, pues, fue primero en Alemania y Austria donde se desarrolló a finales del s. XIX un fuerte debate sobre la estructura y la función de las emociones. Este debate fue importado a EUA y a Reino Unido por algunos estudiantes ingleses y americanos que se doctoraron en centroeuropa (como William James y Edward B. Titchener). Sorprenderá al lector observar como muchas de las tesis actuales ya han sido defendidas anteriormente en la historia de la filosofía. Con esta segunda labor, de carácter historiográfico, quiero acabar con el difundido prejuicio de que hasta su descubrimiento por parte la filosofía analítica las emociones habían sido

1 En este artículo voy a usar los términos emoción y sentimiento como sinónimos. En un sentido estricto, sin embargo, podría trazarse la siguiente diferencia conceptual. El término sentimiento se usa para designar todo aquello que es sentido. Así hay muchos tipos de sentimientos: sentimientos religiosos como la beatitud, existenciales como una envidia existencial, sentimientos estéticos como el goce estético, sentimientos de valor como el asco, sentimientos puramente sensibles como el placer, etc. El término emoción se reserva para un tipo especial de sentimiento que muestra tanto una cualidad sensible como una referencia intencional. Emociones en el sentido estricto de esta palabra son el asco, la vergüenza o la alegría. Para una diferenciación más detallada $c f$. Vendrell Ferran, Í. 2008. 
descuidadas por la historia de la filosofía. Finalmente, voy a mostrar aquellos trechos fundamentales que toda definición de las emociones debe tener en cuenta para adecuarse plenamente a este fenómeno.

La cuestión principal a la que todas las teorías de las emociones deben dar respuesta es la pregunta por lo que define a una emoción como tal, i. e., qué es lo que hace de una emoción una emoción concreta y no otra. Por poner un ejemplo ¿qué es lo que hace del asco, asco, y no envidia, vergüenza o amor? Esta es la pregunta por los elementos constitutivos y definitorios de las emociones. A esta pregunta se han ensayado y siguen ensayándose las más diversas respuestas. Ahora bien, a pesar de que cada una de las teorías contemporáneas es por su especificidad única y cualquier subsunción de estas teorías en grupos implique un cierto riesgo de simplificación y reduccionismo, me parece necesario elaborar aquí una especie de cartografía de las principales líneas de investigación.

En la filosofía contemporánea de las emociones se dejan reconocer principalmente dos tipos de respuesta muy diferentes entre sí. Un primer tipo de respuesta se centra en los aspectos cualitativos e intenta definir las emociones por el modo fenoménico en el que son sentidas corporalmente. A este tipo de teorías se las denomina «Teorías del sentir». El segundo tipo de respuesta se centra en los aspectos cognitivos de las emociones y, por ello, reciben el nombre de «Teorías cognitivistas». Durante las últimas décadas del siglo XX se llegó a presentar ambas teorías como contra-polos teóricos entre los cuales no había reconciliación posible (P. Griffiths 1998, p. 3). La mayoría de autores analíticos desde 1970 hasta finales de los 90 abogaban por teorías de carácter cognitivista resaltando la relación entre emociones y actos de pensamiento y dejando de lado el aspecto cualitativo. Sin embargo, a inicios del año 2000 y como reacción a algunas posiciones cognitivistas extremas, algunos autores empezaron a centrarse en los aspectos cualitativos afirmando que ninguna teoría de las emociones daba cuenta completa de este fenómeno si dejaba de lado el momento de afectación corporal. Ha sido precisamente a partir de estas posiciones críticas que se han esbozado nuevas posiciones las cuales intentan tomar en consideración tanto aspectos cognitivos como aspectos cualitativos. La tesis de fondo implícita en estas nuevas propuestas y que aquí queremos formular de un modo explícito es que las teorías del sentir y las teorías cognitivistas no son polos opuestos, sino complementarios, pues cada una de ellas trata un aspecto distinto de la experiencia emocional. En lo que sigue voy a precisar ambas líneas teóricas mostrando el núcleo argumentativo y el desarrollo histórico de cada una de ellas. 


\section{LAS TEORÍAS DEL SENTIR: DECADENCIA Y AUGE EN CONTEXTO HISTÓRICO}

Centrémonos primero en las teorías del sentir. Las teorías del sentir definen a las emociones como un tipo especial de vivencia que se caracteriza por su momento corporal. El modo cualitativo en el que son sentidas corporalmente resulta desde este punto de vista esencial y definitorio de la experiencia emocional. Tener una emoción significa sentir esta emoción de un modo cualitativo determinado. Obsérvese que en este marco teórico las emociones tienen que ser esencialmente también experiencias o vivencias conscientes, pues este sentirlas corporalmente debe ir acompañado de algún tipo de conciencia. ${ }^{2}$ En el marco de las teorías del sentir, son las cualidades sentidas las que cumplen con la función de diferenciar una emoción de otra y, por tanto, actúan como principio individuador. El hecho de que el asco se sienta como una aversión y deseo de distanciarse del objeto, que afecta a la zona del estómago y de las vías digestivas, es lo que diferencia a esta emoción de la envidia que se siente como una angostura en el pecho y viene acompañada de tendencias destructivas hacia su objeto, de la vergüenza que es cualitativamente sentida como un quererse hacer pequeño e impulsa al abandono de la situación desencadenante de esta emoción, y del amor que es sentido como una amplitud y calor en el pecho y viene acompañado de las más diversas tendencias positivas y favorables hacia su objeto. En este paradigma las emociones son asimiladas al modelo de la percepción, especialmente a la percepción de los cambios corporales. Así la vergüenza consistiría en la percepción del rubor en las mejillas, del calor en la zona de la cabeza, de las palpitaciones, del sudor, etc.

Históricamente las teorías del sentir tienen su origen en la definición cartesiana de las emociones como «perceptions qu on rapporte seulement à l'âme» (R. Descartes 2006), según la cual las emociones se definen por el modo cualitativo específico en el que son experienciadas. La definición cartesiana influyó el panorama filosófico de tal manera que hasta finales del S. XIX casi todas las teorías de las emociones eran teorías del sentir. Wilhelm Wundt, fundador de la psicología experimental alemana, postulaba una definición de las emociones claramente en línea con la definición cartesiana. Frente a los «sentimientos» (Gefühle) que de por sí son pasajeros, poco intensos y sin relación de continuidad con estados pasados y futuros del organismo, se hallan según Wundt los «afectos» (Affekte) -o en nuestra terminología actual las «emociones»- que no son más que conglomerados de «sentimientos» dotados de unidad e intensidad (W. Wundt 1920, p. 204). Las emociones, según Wundt, se oscilan entre los polos opuestos de tres dimensiones cualitativas diferentes: excitación-tranquilidad,

2 De Sousa también ha hecho hincapié en este rasgo de las teorías del sentir (R. De Sousa 1987). 
tensión-relajamiento y placer-desplacer. Para las emociones la dimensión más importante y determinante es la del placer y desplacer, ya que indica si una emoción es sentida como agradable o desagradable. También los discípulos de Wundt que formaron en Alemania varias escuelas psicológicas como Oswald Külpe y que ejercieron mucha influencia sobre los teóricos de la psicología de la Gestalt formulaban teorías parecidas. Los otros discípulos de Wundt que emigraron a América o que eran americanos siguieron también -aunque con diversas críticas- esta definición de las emociones a partir de sus aspectos cualitativos. El inglés Edward B. Titchener, que tras doctorarse con Wundt en Alemania emigró a Estados Unidos y se convirtió en el padre de la Psicología estructural americana, se basó en la definición de Wundt para elaborar su propia teoría, aunque para Titchener sólo la dimensión «placer-desplacer» podría considerarse como válida (E. B. Titchener 1980, p. 226). Fue precisamente William James, uno de los discípulos americanos de Wundt más destacados, quien formuló a finales del s. XIX la versión más conocida de las teorías del sentir. James afirmaba «that the bodily changes follow directly the perception of the exciting fact, and that our feeling of the same changes as they occur IS the emotion»(W. James 1967, p. 13). Según esta definición una emoción consiste en la apercepción de los cambios corporales producidos a partir de un estímulo. Una concepción de las emociones bastante similar fue desarrollada curiosamente un año antes que James por el médico danés Carl Lange. Lange afirmaba: «Take away the bodily symptoms from a frightened individual; let his pulse beat calmly, his look be firm, his color normal, his movements quick and sure, his speech strong, his thoughts clear; and what remains of his fear?» (K. Lange 1967, p. 66). La respuesta es clara: Sin los cambios corporales, no hay emoción. Las teorías del sentir fueron de tal importancia que incluso las primeras tesis de Freud sobre las emociones son de este tipo, como queda patente en su artículo de 1915 «Über das Unbewusste», donde afirma que a la esencia de los afectos pertenece el ser sentidos, el ser percibidos y, por consiguiente, el ser conscientes (S. Freud 2005, 117-155). Más tarde Freud cambiará esta tesis y postulará -como lo hace el psicoanálisis actual (M. Stocker 1996, pp. 21, 23) - que los afectos pueden ser inconscientes.

¿Por qué fueron tan importantes las teorías del sentir hasta finales del S. XIX? Para comprender el auge de este paradigma debemos tener en cuenta que el modelo de la mente dominante hasta finales del s. XIX era el modelo cartesiano. El modelo cartesiano propició el uso de la introspección como método de estudio. La introspección consistía en la observación y descripción detallada de los fenómenos psíquicos que realizaban los «introspectores», personas entrenadas para observar y describir con gran detalle la propia vida anímica. Con tal metodología resultaba fácil investigar las percepciones, por ejemplo, de colores, las sensaciones producidas por tonos o olores y las emociones, 
aunque otros fenómenos como los juicios y otros actos de pensamiento escapaban al estudio introspectivo. Buen testigo de ello es que Külpe, el fundador de la Escuela de Würzburg, se queja de que se ha dedicado poca atención a los procesos de pensamiento y señala como motivo el hecho de que la sensación, la percepción y las emociones resultan más fáciles de investigar con la introspección (O. Külpe 1912). En el marco del cartesianismo, pues, se centró la atención en aquellos fenómenos que más fáciles resultaban de estudiar con la introspección y se empezó por diferenciar a las sensaciones de otros fenómenos semejantes como las percepciones y los sentimientos. ${ }^{3}$ Bajo estas condiciones las emociones aparecían siempre tratadas desde el punto de vista cualitativo en el que son corporalmente sentidas y, por ello, en la filosofía, y en la por aquel entonces incipiente psicología, las teorías del sentir resultaron ser dominantes, una tendencia que prosiguió hasta bien entrado el s. XX.

Ahora bien, entrados ya en el s. XX las teorías del sentir dejaron de estar en boga y empezaron a formularse teorías de corte cognitivista, en las que se intentaba definir las emociones no a partir del modo en que son sentidas, sino a partir de los aspectos de pensamiento implicados en ellas. Las teorías del sentir entraron en el contexto de la filosofía analítica en una fase de decadencia que duró hasta finales de los años 90, para después empezar a ser tomadas de nuevo en consideración como reacción en contra aquellas formas extremas de cognitivimo que dejaban de lado los aspectos corporales. Sin embargo, los autores contemporáneos que defienden teorías del sentir también hacen hincapié en los aspectos cognitivos de las emociones, sacando partido a aquellos aspectos de lo emocional que el cognitivismo acertadamente ha puesto de relieve. Las posiciones analíticas actuales que conciben lo cualitativo como definitorio de lo emocional se pueden dividir en los siguientes grupos:

A. Teorías del sentir tipo A: Este tipo de teorías del sentir asimila a las emociones al modelo de la percepción o la sensación. Entre estos teóricos destacamos a Michael Stocker, el cual ha concebido este sentir cualitativo como «psychic feelings» (M. Stocker 1987, pp. 59-69). Sabine Döring que junto a Christopher Peacocke afirma que las emociones son un tipo especial de percepciones que tienen un componente de sensación y que se dirigen intencionalmente a un contenido representacional: «eine Emotion ist ein aktualer, bewusster Zustand mit einem bestimmten Affekt und einer bestimmten Art von intentionalem, nämlich repräsentationalem Inhalt» (S. Döring y C. Peacocke 2002, p. 95). La última formulación de Döring de las emociones como «affective perceptions»

3 De hecho, los términos sensación, percepción y emoción se usaban a menudo indistintamente. En el ámbito lingüístico alemán, por ejemplo, el término «Gefühl» daba cuenta de los tres (M. Frank 2002, p. 20). 
también cae bajo esta rúbrica. ${ }^{4} \mathrm{Y}$ muy especialmente a Jesse Prinz que se ha esforzado por defender una teoría del sentir basándose en la definición de James de que las emociones son una forma de percepción. Prinz afirma que las emociones surgen a partir de la percepción de estados corporales provocados por cambios en el organismo y el entorno: «emotion is a form of perception. We detect changes in organism/environmental relations by monitoring bodily states» (J. Prinz 2004). Todas estas posiciones intentan explicar a las emociones recurriendo al modelo de la percepción, sin olvidar los aspectos cognitivos de las mismas.

B. Teorías del sentir Tipo B: Este segundo tipo de teorías del sentir no trabaja con el modelo de la percepción, sino con el modelo del juicio. A pesar de que se destaque el papel del juicio en las emociones, estos teóricos no olvidan que las emociones son en primer término fenómenos corporales. Entre estos teóricos destaca hoy en día Benett Helm. Helm entiende las emociones como «felt evaluations», es decir, como evaluaciones sentidas. Las emociones son según este autor un fenómeno irreducilble, en el que las evaluaciones o juicios de valor juegan un papel importante y que en razón de su ser sentidas corporalmente se mueven entre las dimensiones del placer y del desplacer. Este aspecto cualitativo lo concibe Helm -al igual que antes lo hiciera Wundt y Tichener- como «pleasure or pain», i.e., placer o desplacer, rúbricas bajo las cuales cae según esta teoría toda emoción (B. Helm 2002, p. 13-30).

C. Teorías del sentir Tipo C: Este tipo de teorías concibe a las emociones como un fenómeno sui generis no asimilable al modelo de la percepción ni mucho menos al modelo del juicio. El más destacado dentro de este marco es Peter Goldie que define a las emociones como un «feeling towards» (P. Goldie 2002), es decir como un «sentir hacia», y con ello resalta no sólo el papel jugado por los aspectos cualitativos sino también por lo cognitivos.

Conviene mencionar aquí que, fuera del marco de la filosofía analítica, la corriente neo-fenomenológica siguió postulando, incluso en los años en los que el cognitivismo estaba más en boga que el aspecto cualitativo es definitorio de las emociones. Basándose en la idea homérica de que las emociones son fuerzas que se apoderan de los seres humanos, elabora Hermann Schmitz su teoría resaltando que las emociones nos envuelven, se hacen dueñas de personas y situaciones y son sentidas corporalmente de un modo característico. Las emociones -afirma Schmitz- son atmósferas espaciales, pero que no tienen un lugar fijo: «räumlich, aber ortlos, ergossene Atmosphären» (H. Schmitz 1998, p. 22; en el mismo sentido: Schmitz 1981, p. 152). Hilge Landweer en la misma tónica sostiene la tesis de que las emociones son «acontecimientos»

4 Cf. para más autores Slaby, J., (2008): Gefühl und Weltbezug. Paderborn: Mentis. 
(Widerfahrnisse) (H. Landweer 2004, p. 485) y afirma que las emociones se definen por una afectación corporal (leibliche Betroffenheit) la cual designa la cualidad intrínseca con la que es sentida cada una de ellas. Estos autores trabajan con la diferencia fenomenológica -ignorada hasta el día de hoy en la filosofía analítica ${ }^{5}$ - entre el cuerpo físico (Körper), que hace referencia a nuestro cuerpo extenso en un espacio determinado, y el cuerpo vivo (Leib), que hace referencia a aquello que se puede sentir de nuestro cuerpo y sus proximidades sin uso de ninguno de los cinco sentidos. El cuerpo vivo es un lugar absoluto que posee su propia dinámica y que no puede reducirse a cuerpo físico. Se trata no de nuestro cuerpo físico ni de como éste nos es dado en la percepción externa, sino de nuestro cuerpo tal y como lo sentimos en un momento determinado. A partir de esta diferencia definen a la corporalidad como esencial de las emociones en el sentido de que cada emoción se caracteriza por una manera peculiar de ser vivenciada corporalmente.

¿Cuáles son los argumentos de los defensores de las teorías del sentir? La principal línea argumentativa consiste en apelar a la experiencia inmediata y cotidiana, según la cual las emociones nos afectan corporalmente. A menudo se ha recurrido para argumentar en favor de esta teoría a un experimento mental al estilo argumentativo de James y Lange. El experimento consiste en imaginarnos que tenemos una emoción y entonces intentar hacer abstracción de sus momentos corporales. Por poner un ejemplo, si cuando estamos furiosos hacemos abstracción del calor que sentimos, la palpitación acelerada, la subida de sangre a la cabeza, la angostura en el pecho, la sensación de estar a punto de explotar, los movimientos que aspiran a la destrucción del objeto de nuestra ira o hacia un objeto que simbólicamente pueda representarlo, etc., entonces -siguiendo este argumento- no nos queda nada de la emoción, ya que la emoción misma consiste precisamente en este momento corporal. El otro argumento en favor de las teorías del sentir se basa en el hecho de que adoptando un determinado estado corporal -por medio de drogas u otras sustancias o simplemente adoptando la expresión corporal de la una emoción- se puede originar la emoción que corresponde a ese estado corporal.

A pesar de la evidencia de la experiencia inmediata y a pesar de estos dos argumentos, las teorías del sentir en todas sus variedades deben hacer frente a una serie de objeciones de las que vamos a destacar las siguientes:

Problemático resulta, en primer lugar, el que dentro del paradigma del sentir a menudo se intenta reducir a las emociones a percepciones de los cambios corporales y a consecuencia de ello se roba la originariedad al fenómeno emocional.

5 Una excepción sería Matthew Ratcliffe el cual basándose en la fenomenología de Heidegger y de Merleau-Ponty trata el aspecto de la corporalidad de las emociones (M. Ratcliffe 2005). 
Otro problema de estas teorías es que si bien los aspectos corporales resultan esenciales para las emociones, éstos no bastan para diferenciar una emoción de otra. Pues a veces son demasiado difusos, otras veces son comunes a emociones diferentes y otras son comunes a emociones y estados que no son emocionales. Pues, uno puede ruborizarse por vergüienza, pero también por furia, y uno puede estar pálido y tener sudor fría por miedo, pero también a causa de la fiebre. La caracterización de una emoción sólo a partir de los cambios corporales no es, pues, suficiente.

Cuestionable resulta, además, la tesis de que los cambios corporales son siempre conscientemente sentidos. Pues parece no contradecir los datos de nuestra experiencia el que podamos experimentar cambios corporales que no vienen acompañados de una conciencia reflexiva. Por ejemplo, uno puede temblar de miedo y, a la vez, no darse cuenta de que está temblando.

El cuarto problema es que la mayoría de las teorías del sentir no postulan ningún vínculo esencial entre la emoción y su objeto. En el marco de estas teorías, las emociones parecen estar vinculadas con sus objetos de un modo puramente casual. Sin embargo, como ya hizo patente Anthony Kenny, las emociones poseen dos tipos de objetos: el objeto material y el objeto formal. Recurriendo a un ejemplo, supongamos que alguien siente miedo ante una tormenta. La tormenta es el objeto material de la emoción del miedo; el objeto formal es la cualidad de lo peligroso, lo temible, lo amenazante que en estos momentos se da en el objeto material de la tormenta. Mientras el objeto material del miedo puede cambiar cada vez que sentimos esta emoción (se puede temer a una tormenta, pero también a una persona, una situación concreta, etc.) y está determinado por factores socioculturales e históricos; el objeto formal del miedo siempre es el mismo lo peligroso, lo temible o lo amenazante. Kenny escribe: «The formal object of ping is the object under that description which must apply to it if it is to be possible to $\phi$ it. If only what is $\mathrm{P}$ can be $\phi \mathrm{d}$, then «thing which is $\mathrm{P}$ » gives the formal object of фing». (A. Kenny 1963, p. 192) Según esta tesis cada emoción tiene su objeto formal propio con el que está vinculada de un modo esencial: la emoción a sólo se puede dirigir hacia el objeto A, la emoción b sólo hacia el objeto B. Así el objeto formal del asco es lo asqueroso, el de la vergüenza es lo vergonzoso, el miedo lo temible, etc., y esta relación jamás puede cambiarse. Este vínculo especial de las emociones con sus objetos formales revela que las emociones no son simplemente estados corporales, sino que se dirigen a una determinada suerte de objetos formales con los cuales están en una relación de necesidad. Esta relación viene a conocerse con el nombre de intencionalidad. Una implicación importante de esta tesis de la intencionalidad de las emociones formulada en la filosofía analítica por Kenny, pero expresada ya a finales del s. XIX por Brentano y a principios del s. XX por Husserl, Scheler, Stein y los primeros fenomenólogos, es que hay 
para las emociones condiciones de verdad. Dado que cada emoción tiene objetos formales que le son apropiados, si una emoción se dirige a un objeto formal que no le es propio, la emoción es inapropiada. Esto sucedería cuando se siente, por ejemplo, miedo de lo vergonzoso o asco de lo temible. Esta tesis realza las semejanzas entre emociones y juicios, ya que estos también tienen condiciones de verdad y pueden ser correctos, verdaderos o falsos, incorrectos. De hecho Kenny afirma que la intencionalidad de las emociones se deriva precisamente de los juicios que ellas tienen por base. Con esta tesis se diferencia, por un lado, de las posiciones de Brentano y los fenomenólogos que reconocen a las emociones una intencionalidad sui generis no derivada de los juicios, $\mathrm{y}$, por otro lado, de las teorías del sentir radicales que obvian a menudo el hecho de que las emociones sean intencionales (los teóricos del sentir actuales sí atienden a este hecho de la intencionalidad).

La quinta objeción es que si -como las teorías del sentir afirman- las emociones son un sentir los cambios corporales resulta inexplicable en este marco como puede darse más de una emoción a la vez, ya que en un mismo momento ni pueden sentirse cualidades completamente diferentes ni podemos hacernos conscientes de todos los cambios cualitativos que experimentamos. Estas teorías no pueden explicar como es posible sentir al mismo tiempo, vergüenza y temor, por poner un ejemplo. Pues, mientras la vergüenza se define por el rubor y el calor, el temor se define por la palidez y el frío. Tampoco explican estas teorías como es posible que estemos furiosos con una persona a la cual amamos, pues parece que la cualidad de la ira en estos instantes ofusca casi por completo la cualidad fenoménica del amor y, sin embargo, podemos amar a una persona con la cual estamos muy enfadados.

Relacionado con lo anterior está aún el problema de que las teorías del sentir no pueden explicar como es posible tener meta-emociones, i.e., tener una emoción sobre una emoción. Por ejemplo, cuando siento vergüenza y me enojo por ello, este enojo es una meta-emoción, porque es una emoción sobre la emoción de la vergüenza, o cuando siento envidia y me avergüenzo por ello, la vergüenza es aquí una meta-emoción sobre la envidia, o cuando siento vergüenza por sentir vergüenza, la vergüenza sobre la vergüenza es una meta-emoción. Dejando de lado este último caso, en el que meta-emoción y emoción coinciden, por lo general meta-emociones y emociones son diferentes, de modo que corporalmente son sentidas de modo también diferente, lo que resulta inexplicable para las teorías del sentir.

La última gran objeción a estas teorías concierne la tesis de que las emociones son siempre sentidas, conscientes. Esta tesis es muy problemática pues no toma en consideración la existencia de emociones subconscientes que pueden influenciar nuestro pensar y actuar a largo plazo sin que tengamos la menor sospecha de ellas. Por ejemplo, podemos odiar a una persona durante años, sin 
que este odio se haga presente, se manifieste o sea sentido de modo consciente. Como el psicoanálisis ha demostrado, este odio puede existir como emoción de modo latente y manifestarse sólo en ocasiones muy puntuales. Las teorías del sentir con su tesis de que las emociones son siempre conscientes no toman en consideración esta posibilidad, reducen el término emoción a estados actuales y conscientes (de hecho a las emociones que no son conscientes no las denominan emociones, sino simplemente disposiciones) y, con ello, olvidan gran parte de nuestra vida afectiva que tiene lugar a un nivel subconsciente.

A pesar de estos puntos críticos la gran virtud de las teorías del sentir consiste en oponerse a los cognitivismos extremos que -en especial durante los últimos decenios del siglo $\mathrm{XX}$ - han tendido a olvidar el cuerpo y los aspectos cualitativos de las emociones. Las emociones por esencia nos afectan corporalmente con una cualidad específica de ser sentidas y lo hacen de un modo en el que jamás un mero juicio, una percepción o un recuerdo -si no van acompañados de una emoción- pueden hacerlo.

\section{LAS TEORÍAS COGNITIVISTAS: DESARROLLO HISTÓRICO Y TIPOLOGÍAS ACTUALES}

La otra gran línea interpretativa de las emociones viene constituida por las teorías cognitivistas. Como William Lyons ha señalado el cognitivismo define las emociones por sus aspectos cognitivos (W. Lyons 1980, p. 33). La influencia de factores de pensamiento para las emociones ha sido reconocida desde muy antiguo: Descartes -a pesar de defender una teoría del sentir-reconocía que los aspectos cognitivos influyen en las emociones y Hume definió a las emociones en base de los juicios implicados en ellas. Ahora bien, sólo en el s. XX se ha establecido ampliamente la tendencia a considerar los aspectos cognitivos como el factor definitorio exclusivo de las emociones. Las teorías cognitivistas han dominado el panorama de la filosofía de las emociones desde la segunda mitad del S. XX hasta la actualidad invirtiendo la tendencia histórica desde Descartes hasta inicios del S.XX en que preponderaban las teorías del sentir.

El auge del cognitivismo durante este período se explica por la confluencia de dos factores históricos. Por un lado, está relacionado con el hecho de que desde finales del s. XIX se empieza a ver la conciencia no ya como un espacio cerrado con contenidos capaces de ser aprehendidos por medio de la introspección, sino como acto que intenciona objetos del mundo y que explica nuestra conducta racional. Este cambio fue propiciado por la definición de la conciencia como intencional por parte de Francisco Brentano (F. Brentano 1924, p.125). Por otro lado, está vinculado con un nuevo interés por la función adaptativa al entorno que cumple la conciencia. Este factor vino propiciado por la concepción de Darwin y de los evolucionismos que consideraban la adaptación como una 
de las funciones fundamentales de todo ser vivo. A raíz de estas dos tendencias históricas se centra el interés en el comportamiento y la conciencia como reguladora del mismo. Las emociones en este nuevo paradigma no son investigadas en sus aspectos cualitativos por medio de la introspección, sino a partir de su supuesta función adaptativa y reguladora del comportamiento. El interés por las emociones en función de su capacidad de aportarnos información sobre el mundo y de regular el comportamiento racional se fue agudizando según mi parecer a partir de los años 50 y alcanzó su clímax en los años 80 y 90 del s. XX. Precisamente en este período proliferan las teorías que se centran en los aspectos cognitivos de las emociones olvidando por completo los aspectos cualitativos y fenoménicos de lo afectivo.

Según el esquema que voy a proponer aquí existen dos tipos generales de teorías cognitivistas de las emociones y cada uno de estos tipos puede presentarse en dos versiones, una no reduccionista y la otra reduccionista. ${ }^{6}$

A. Cognitivismo Tipo A: El cognitivismo es ententido en esta variante en sentido amplio. Cognitivismo designa aquí el hecho de que las emociones se basan en actos de pensamiento, es decir, que las emociones precisan de bases cognitivas para tener lugar. Ahora bien, según el tipo de base cognitiva se distinguen dos clases de cognitivismo del tipo A.

A. 1. La versión no reduccionista de este tipo de cognitivismo afirma que las bases cognitivas de las emociones pueden ser de muy diversos tipos: percepciones, juicios, suposiciones, fantasías, recuerdos, etc. Por ejemplo, para sentir asco, debo tener primero una percepción de un objeto; o para alegrarme de un hecho debo haber juzgado un hecho como positivo; o para sentir miedo de un monstruo debo haber fantaseado este monstruo; o para sentir arrepentimiento de un acto del pasado, debo recordar lo que hice. Este tipo de cognitivismo no ejerce un papel dominante en el panorama actual, pues muchos autores analíticos defienden la tesis de que los únicos actos que funcionan como bases cognitivas de las emociones son los juicios.

Las raíces históricas de este cognitivismo no reductivo hay que buscarlas a inicios del S. XX en la fenomenología. Así encontramos defendiendo esta tesis a Alexander Pfänder y a Edith Stein. Según estos autores las emociones se pueden basar en juicios, percepciones o fantasías (A. Pfänder 1922, p.16; E. Stein 1917, p. 112). Entre los autores actuales que defienden esta tesis encontramos a Stocker (Stocker 1987, pp. 59-69) y Patricia Greenspans (P. Greenspan 1988, pp. 223-250) que afirman que junto a los juicios, también la fantasía puede actuar como base de lo emocional; a Jon Elster que reconoce junto a los juicios tam-

6 Tomo esta terminología de Jan Slaby, aunque utilizo estos términos en un sentido diferente (J. Slaby 2004, pp.50-85). 
bién a las percepciones como bases cognitivas (J. Elster 1999, p. 250); a Kevin Mulligan que afirma que las bases de las emociones son percepciones, juicios y recuerdos (K. Mulligan 1998, p.168) y a Goldie que afirma que las bases son fantasías, juicios y percepciones (P. Goldie 2002, p. 45).

Estos autores argumentan que aquellos que consideran exclusivamente a los juicios como bases de las emociones -es decir, la gran mayoría de filósofos analíticos- dejan sin explicación gran parte de la vida afectiva. Como es el caso del asco que se siente al oler algo, lo que no implica un juicio, sino una percepción, o el miedo que se siente al imaginar un mostruo, que tampoco se basa en un juicio, sino en una fantasía. El cognitivismo no reduccionista al ampliar aquello que puede funcionar como base de las emociones está más acorde con la variedad de experiencias emocionales y es, además, compatible con la idea básica de las teorías del sentir según la cual a las emociones les es propia una dimensión cualitativa.

A.2. La versión reduccionista de este tipo de cognitivismo afirma que las únicas bases cognitivas de las emociones son los juicios o actos parecidos a los juicios como las suposiciones o las creencias. Es decir, según este tipo de cognitivismo en su versión reduccionista, sentir asco implica que he antes he juzgado algo como asqueroso, sentir alegría implica que antes he juzgado algo como alegre, sentir miedo implica que antes he juzgado algo como terrible y sentir arrepentimiento implica haber juzgado algo como digno de arrepentimiento. Al juicio que sirve de bases a las emociones se lo denomina juicio identificatorio porque es el que identifica, es decir, individualiza a las emociones como tales (G. Taylor 1985, p. 3). El juicio identificatorio cabe distinguirlo del juicio explicatorio que sería el juicio que nos proporcionaría la explicación causal del porqué algo es juzgado como asqueroso, alegre, terrible o digno de arrepentimiento. Por ejemplo, si siento miedo de una araña el juicio identificatorio sería el juicio «la araña es peligrosa», mientras que el juicio explicatorio sería la razón por la cual se ha juzgado a la araña como tal, i.e., el juicio: «algunas arañas son venenosas». Esta versión del cognitivismo está muy extendida en la filosofía analítica.

Los antecedentes históricos de esta tesis radican ya en Hume que afirma que las emociones se basan siempre en juicios (M. Frank 2002, p. 20). También en algunos autores que precedieron a la fenomenología como Brentano y Stumpf. Para Brentano había tres tipos de actos intencionales: las representaciones que podían proceder de la percepción o de la fantasía, los juicios y los actos que él llamaba de amor y de odio, y que en realidad era una clase que incluía tanto actos de voluntad como emociones. Según la tesis de Brentano -que al igual que Kenny decenios más tarde, desarrolló basándose en la escolástica- las emociones como actos de la tercera clase implicaban actos de la primera y de la segunda clase, i.e., se basaban en representaciones y en juicios (F. Brentano 
1921, p. 16; F. Brentano 1924, p. 35). Uno de los discípulos de Brentano, fue el maestro de Husserl Carl Stumpf. Stumpf al igual que Brentano afirmaba que las emociones se dirigen a estados de cosas que deben ser objeto de un juicio: «Bei allen Gefühlszuständen, die zweifellos und im eigentlichen Sinne zu den Affekten gezählt werden, sagen wir, dass sie sich auf einen Sachverhalt beziehen, über den wir uns freuen, ärgern, erzürnen, vor dem wir uns fürchten usw. Das heißt: der Affekt gründet sich auf ein Urteil» (C. Stumpf 1928, pp. 2-3, también en p. XIII). Es decir, que las emociones se fundan en juicios. En la filosofía analítica la tesis de que las emociones se basan únicamente en juicios aparece por primera vez en el libro de Anthony Kenny Action, Emotion and Will (A. Kenny 1963, p. 195). Ahora bien, a pesar de la similitud con Brentano, mientras éste reconoce una intencionalidad propia a las emociones, Kenny hace derivar la intencionalidad de las emociones de los juicios. La tesis de Kenny será punto de partida y fuente de inspiración de posteriores desarrollos en la filosofía analítica de las emociones. Así, por ejemplo, la encontramos defendida en los textos de Gabriele Taylor (G. Taylor 1985) y en O. Harvey Green (O.H. Green 1992), por citar algunos autores.

La tesis defendida por estos autores es que las emociones se basan siempre en juicios sobre objetos, estados de cosa, personas, etc., y que su intencionalidad se debe precisamente a que se basan en juicios. Su idea es que no nos podemos alegrar de algo sin antes haberlo juzgado como positivo, ni entristecernos por algo sin antes haber formulado un juicio negativo sobre ello. El principal argumento de estas teorías es que entre emociones y juicios existe una relación de dependencia. Si cambiamos el juicio sobre el que se basa una emoción, la emoción también cambia. Si, por ejemplo, creo que alguien me ha criticado y juzgo a esta critica como negativa para mi persona, estaré enfadada con él. Si en cambio después descubro que esa creencia era falsa, mi enfado desaparecerá.

Esta versión del cognitivismo se enfrenta con algunas objeciones. En primer lugar, implica una «sobre-intelectualización» de las emociones, pues presupone que toda emoción viene precedida de un juicio (creencia o suposición) sobre el objeto, con lo cual se quita espontaneidad a la vida emocional. Esta sobre-intelectualización, sin embargo, parece contradicha por la rapidez con la que muchas veces las emociones tienen lugar. Por ello, parece más adecuado tomar como base de las emociones no sólo a los juicios (y actos afines), sino también a percepciones, recuerdos, fantasías, etc. Esta sobre-intelectualización queda también comprometida por el hecho mismo de que, a menudo, los juicios tienen lugar sólo después de sentir una emoción. Supongamos el caso de que ante una situación determinada sentimos indignación, y sólo después de haber tenido este sentimiento juzgamos a la situación como injusta. O supongamos que sentimos una extrañeza ante un hecho concreto y sólo con posterioridad y en base de este sentimiento pronunciamos un juicio sobre tal hecho. En este 
sentido resulta cierto no sólo que las emociones no precisan de juicios para tener lugar, sino que ellas también pueden ser base de juicios de valor y a su vez incluso justificarlos.

Otro punto problemático de esta tesis reduccionista es que establece una brecha insoslayable entre las emociones de las personas con capacidad de juzgar y las emociones de los animales, de los niños y de personas con menos capacidades cognitivas. Pues implica esto, que sólo pueden tenerse emociones si existe la capacidad para actos elaborados de pensamiento como son los juicios, y queda sin explicar qué es lo que sienten los seres con menores capacidades intelectuales (R. Wollheim 1999).

Finalmente resulta problemático para los partidarios de esta tesis explicar el funcionamiento de algunas emociones que no se basan en juicios como el asco basado en percepciones como olores, tonos, sabores, etc. y algunos tipos de miedo basado en fantasías. Así, pues, podemos concluir que si bien resulta cierto que algunas emociones se basan en juicios, este hecho no se puede universalizar, ya que muchas emociones quedan sin explicar si no se toman como base de las emociones un abanico más amplio de actos.

B. Cognitivismo Tipo B: El segundo tipo de cognitivismo sostiene la tesis de que las emociones tienen una función cognitiva que consiste en aportarnos información sobre el mundo. Este cognitivismo se presenta también en dos versiones una no reduccionista y una de reduccionista, que vamos a analizar en detalle a continuación.

B.1. La versión no reduccionista afirma que las emociones son un fenómeno sui generis que nos aportan información sobre el mundo. Las emociones se encargarían de que el mundo no se nos presente como un terreno neutral, sino como un relieve en el que algunas cualidades se destacan mientras que otras nos pasan desapercibidas y nos guiarían con ello en nuestra acción. Estas cualidades especiales de nuestro entorno que las emociones se encargan de captar, aprehender, percibir reciben el nombre de «cualidades axiológicas» o valores. Los valores son, según esta versión del cognitivismo, los objetos formales de las emociones. El asco tiene por objeto formal la cualidad axiológica de lo asqueroso, el miedo la de lo amenazante, etc. Estas teorías operan con una analogía entre emoción y percepción y afirman que al igual que en la percepción captamos los colores como cualidades de las cosas, en un acto del sentir captaríamos cualidades axiológicas en determinados los objetos, personas, situaciones, estados de cosas, etc. En virtud de esta analogía con la percepción, por otra parte, este tipo de cognitivismo es compatible con la ideal principal de las teorías del sentir según la cual las emociones implican una afectación corporal.

Respecto a la relación entre emociones y valores hay en el panorama actual diferentes posiciones (K. Mulligan 1998, K. Mulligan 2004). El emotivismo 
afirma que los valores son proyecciones de nuestros estados afectivos en el mundo, ${ }^{7}$ el constitucionalismo defiende la tesis de que los valores están constituidos social y culturalmente y que las emociones juegan en ello un destacado papel (Stocker 1996) y el realismo ontológico sostiene que los valores existen independientemente de las personas que los puedan aprehender y que este estatus ontológico específico suyo requiere de una epistemología propia para poderlos captar: el «sentir» sería aquí la capacidad que nos haría posible el acceso al mundo de los valores (M. Scheler 1954, C. Tappolet 2000, M. Johnston 2001). De entre todas estas posiciones parece que el realismo ontológico está cobrando fuerza en el debate actual. Respecto a esta capacidad de «sentir» se bifurcan las opiniones. Algunos autores afirman que son el sentir es él mismo una emoción; otros afirman que cabe distinguir estas emociones de los actos del sentir mismos.

Los antecedentes históricos de esta posición según la cual los valores son los objetos de las emociones se hallan ya en Brentano y todos los primeros fenomenólogos. Si bien entre estos estaban las opiniones divididas. Mientras Husserl, Meinong, Stein y Kolnai afirmaban que las emociones captaban los valores (E. Husserl 1992, p. 404; A. Meinong 1968, pp. 130-131; E. Stein 1917, pp. 109-110, 112; A. Kolnai 1974, p. 128, 166); Scheler, Geiger y Ortega y Gasset afirman que las emociones son respuestas a los valores, pero que los valores son captados en actos del sentir (M. Geiger 1974, p. 8; M. Scheler 1954, p. 271; J. Ortega y Gasset 1966, p. 325, 328, 331). Es decir, que el sentir es un acto cognitivo que capta los valores, pero que en sí no es una emoción. La misma división de opiniones la encontramos en el debate actual. Ronald de Sousa afirma que los objetos formales de las emociones son los valores (R. de Sousa 1987) y basándose en él e inspirándose en tesis fenomenológicas afirma Christine Tappolet que las emociones son ellas mismas percepciones de valor: «quand les circontances sont favorables, nos emotions fournissent un accès cognitive fundamental aux valeurs. [...] Nos emotions pourraient donc étre qualifiées de perceptions des valeurs» (C. Tappolet 2000, pp. 8-9). En el mismo sentido Mark Johnston postula la tesis de que las emociones revelan valores «[...] it is because affect can be the disclosure of the appeal of other things and other people that it can have authority in the matter of what we should desire and do. By "the authority of affect" I mean not to refer to its sheer effectiveness as a source of desire or action, but rather to the fact that the presence of the affect can make the desire or action especially intelligible to the agent himself. It can make the desire or act seem apt or fitting in a way that silences any demand for justification [...]. In this way affect is akin to perceptual experience considered

7 Holmer Steinfath nombra a Alfred Ayer como exponente de esta posición. Cf. Steinfath, H. 2002, p. 110. 
more generally.» (M. Johnston 2001, P. 189). Por otro lado, Kevin Mulligan inspirándose en Scheler afirma que el sentir y las emociones son actos diferentes (K. Mulligan 2004).

Este tipo de cognitivismo B.1. se enfrenta a una serie de objeciones. Por un lado, los defensores de la tesis de que las emociones captan valores se enfrentan a una serie de problemas de las cuales destacaremos dos. Primero, las cualidades axiológicas y las emociones no se corresponden una a una. Pues, el mundo se nos puede presentar con infinidad de matices, pero las emociones que podemos llegar a sentir están limitadas a un determinado número. Estas teorías no pueden explicar, por tanto, que tipo de correlación hay entre emoción y valor. Segundo, contradice a estas tesis el hecho de que podemos sentir valores o cualidades axiológicas sin, por ello, sentir emoción alguna. Por ejemplo, puedo captar la tristeza de un cuadro y no por ello ponerme triste, o puedo captar la injusticia de una situación y no por ello indignarme. ${ }^{8}$ Por otro lado, a los defensores de la tesis de que las emociones son diferentes de la función del sentir, si bien se libran de las dos objeciones anteriores, se les puede objetar que postular la existencia de un tipo de capacidad cognitiva llamada «sentir» que en sí no es «sentida», i. e., un sentir que no supone una afectación corporal, tiene algo de contradictorio en sí.

A pesar de estas dificultades obsérvese aquí que esta versión del cognitivismo resulta compatible con el cognitivismo A.1, según el cual las emociones disponen una amplia variedad de bases cognitivas y con la idea de las teorías del sentir, según la cual, las emociones se caracterizan por una cualidad específica en la que son sentidas corporalmente.

B.2. La versión reduccionista afirma que las emociones tienen una función cognitiva porque ellas mismas son cogniciones. El tipo de cognición por excelencia al que se ha pretendido reducir las emociones es el juicio. El primer autor analítico que presentó esta tesis fue Rober Solomon. Para el caso de la vergüenza queda esta tesis ilustrada del modo siguiente: «My shame is my judgement to the effect that I am responsible for an untoward situation or incident» (R. Solomon 1993, p. 126). Con esta tesis quiere Solomon defender una tesis originaria ya de Sartre según la cual las emociones son acciones, i. e., algo que hacemos y que expresa nuestro estar vueltos hacia el mundo, y no algo que simplemente nos sobreviene. La idea que se intenta defender con esta teoría voluntarística es que las emociones son racionales, de que están bajo nuestro control, de que somos responsables por ellas y no meros seres pasivos abandonados a sus veleidades. Otra variante de esta versión reduccionista es la propuesta por Martha Nussbaum según la cual las emociones son un tipo especial de juicios: juicios de valor o evaluaciones. Inspirándose en la concepción estoica de las emociones, sostiene 
esta autora una tesis que ella misma denomina neo-estoicista según la cual: $\ll[\ldots]$ emotions are forms of evaluative judgement that ascribe to certain things and persons outside a person's own control great importance for the person's own flourishing» (M. Nussbaum 2005, p. 22). En la misma línea reduccionista encontramos la Teoría de las emociones de Joel Marks (J. Marks 1982, pp. 227242) o de O. Harvey Green (O. H. Green 1992) según la cual las emociones son combinaciones de juicios y deseos. Esta línea teórica es conocida como «BeliefDesire Theory of emotions» y ampliamente defendida en la filosofía analítica. Otra versión de esta reducción de las emociones a juicios es aquella que intenta explicar las emociones como combinaciones de juicios y otros elementos. La más conocida de ellas es la «Teoría componencial» de Aaron Ben-zéev, según la cual las emociones son un complejo de juicios, cogniciones, sensaciones y motivaciones (A. Ben-ze'ev 2000, p. 49). Tras esta variedad de posiciones se halla la misma tesis fundamental: las emociones son o bien juicios o bien un tipo especial de juicios o bien juicios combinados con otros actos (deseos, sensaciones, etc.). Estos juicios se realizan sobre determinadas situaciones, objetos y estados de cosa del mundo y son lo que define a la emoción como tal.

Estos autores argumentan en favor de su tesis alegando a las analogías entre emociones y juicios. Las emociones pueden volverse a sentir si las evocamos, al igual que los juicios pueden volver a ser formulados. Emociones y juicios parecen tener condiciones de verdad según su adecuación al objeto, Y, además, la información que las emociones nos aportan sobre el mundo es como un juicio que nos proporciona material informativo y nos dice como debemos orientarnos y actuar.

Con todo, y a pesar de las analogías entre emociones y juicios, se trata de dos fenómenos que son de por sí de una naturaleza muy distinta. En primer lugar, hay una diferencia fundamental entre una emoción y un juicio que estas teorías parecen pasar por alto y es que los juicios cuando los formulamos no los sentimos corporalmente, mientras las emociones sí. Afirmando, por tanto, que las emociones son juicios estas teorías no consideran esencial de las emociones el aspecto corporal y su dimensión cualitativa.

En segundo lugar, puede hacerse frente a estas teorías con lo que Peter Goldie ha llamado el «argumento del señor Spock» (P. Goldie 2002, p.50). Según este argumento, podemos imaginar un ser totalmente capaz de formular juicios racionales, y de tener combinaciones de juicios y deseos, sin por ello mostrar la más mínima alteración emocional, como es el caso del personaje de la serie de Star Treck llamado Spock. Dado pues que podemos formular juicios, tener juicios de valor y combinaciones de juicios con deseos, sin por ello tener la más mínima emoción, resulta absurdo reducir las emociones a juicios, interpretarlas como un tipo especial de juicio (juicios de valor o evaluación) o considerarlas conglomerados de juicios y otros elementos. 
Otro problema de estas teorías es que no pueden explicar como son posibles emociones que no se basan en juicios, sino en percepciones, recuerdos o fantasías. Este problema lo comparten con el cognitivismo reduccionista del tipo A.

Una cuarta objeción se deriva del hecho de que no siempre coinciden las emociones y los juicios sobre un mismo objeto (especialmente en los casos de los juicios de valor), como se pone de manifiesto en los casos de fobias. Por ejemplo, puedo emitir una evaluación sobre las arañas de jardín como un animal inofensivo y, sin embargo, sentir miedo hacia ellas o juzgar que volar en avión es seguro y sentir a pesar de ello pánico a usar este medio de transporte. Los teóricos que reducen las emociones a juicios llaman a las emociones que no coinciden con los juicios «emociones irracionales», ya que en su marco teórico son inexplicables. En cambio, si no reducimos las emociones a juicios parece viable la idea de que puede sentirse una emoción basada en una percepción o una fantasía de un objeto que no coincida con un juicio sobre ese mismo objeto y no por ello es la emoción irracional.

La última objeción a estas teorías es la de que cuando tenemos una emoción nuestro yo parece estar de algún modo mucho más involucrado que cuando formulamos un juicio. Parece que en las emociones que sentimos se pone de manifiesto mucho más sobre nuestra persona que en los juicios que emitimos. Enfadarse por un hecho revela quienes somos, nuestro sistema de valores, nuestras experiencias pasadas y expectativas futuras, mientras que formular un juicio sobre un hecho no pone nuestra persona tan al descubierto. David Pugmire ha expresado esta idea con las siguientes palabras: «[...] while agreeing dispassionately that $p$ is indeed a pity involves one to that extent, it does not involve one's mind as a whole in the way that actually sorrowing over $p$ and the fact that $p$ does» (D. Pugmire 2005, p. 16).

Todas estas objeciones hacen inviables las teorías que reducen las emociones a juicios o a juicios de valor o a combinaciones de juicios y otros actos. En el amplio marco de los tipos de cognitivismo las únicos tipos que parecen hacer justicia a la verdadera naturaleza de las emociones son los no reductivos -del tipo A y del tipo B, que además son compatibles entre sí-, ya que estos dan cabida a considerar aspectos cualitativos de lo emocional.

Sin embargo, y a pesar de estas objeciones teóricas, hay que resaltar en este punto de nuestra exposición que gracias a este giro cognitivista la filosofía de las emociones se prestó atención a algunos aspectos de las mismas que debido a la preponderancia de las teorías del sentir hasta el s. XX apenas habían sido las siglos anteriores investigados. Uno de estos aspectos concierne a la vinculación de las emociones con la racionalidad. Tradicionalmente se ha visto a las emociones como lo opuesto a la razón, al pensamiento. Es un mérito de las teorías cognitivistas el haber señalado que las emociones y la razón no son dos elementos opuestos. Pues, por un lado, las emociones se basan en cogniciones y 
a su vez fundan y justifican cogniciones; y, por otro lado, son actos de caracter emocional los que nos aportan información sobre el mundo de modo que no se trata de meras reacciones corporales irracionales.

\section{HACIA UNA DEFINICIÓN DE LAS EMOCIONES}

Las emociones son y, como hemos mostrado, han sido una uno de los centros de interés de la filosofía. Ante la gran variedad de propuestas la pregunta por la definición de las emociones sigue en el debate actual aún por responder. Lo que en este punto resulta claro es que ni las teorías del sentir en su versión radical ni las teorías cognitivistas en sus versiones reduccionistas pueden dar cuenta de la verdadera naturaleza de las emociones. Las propuestas que más parecen adecuarse al fenómeno son más bien aquellas posiciones que definen las emociones a partir de los aspectos corporales y cognitivos. Las emociones - como afirman las teorías del sentir- son un fenómeno al que le es esencial el afectarnos corporalmente y a su vez -como afirma de un modo general el cognitivismo- están vinculadas esencialmente con actos de pensamiento. Una definición adecuada de las emociones debe pues tener en consideración ambos aspectos.

Precisamente desde el año 2000, como ya hemos mencionado en la exposición de las teorías del sentir, se está produciendo un nuevo giro -aún por consolidar- en las teorías de las emociones en el que diversos autores parecen converger en una posición intermedia de este tipo. A modo de reacción en contra los cognitivismos reduccionistas empezaron algunos filósofos a inspirarse en las teorías del sentir que realzan los aspectos cualitativos de las emociones, sin olvidar por ello, aquellos aspectos que el cognitivismo no reductivo había puesto acertadamente de relieve sobre el fenómeno emocional. El primero de estos autores fue Peter Goldie en su libro The Emotions. Peter Goldie define a las emociones con el concepto de «feeling towards», es decir, un «sentir hacia» que comprende tanto el aspecto corporal como el intencional (P. Goldie 2002). Las emociones son, según Goldie, un fenómeno que sentimos corporalmente y que, a su vez, se basa en bases cognitivas, es intencional y nos aportan información sobre el mundo. La publicación de este libro supuso la apertura de una nueva línea de investigación de las emociones por la cual otros autores también han apostado. Así es como Sabine Döring califica a las emociones de «affective perceptions» (percepciones afectivas) y Benett Helms en la misma línea califica a las emociones de «felt evaluations», es decir, «evaluaciones sentidas». Si bien estos autores siguen recalcando de manera evidente el aspecto cognitivo de lo emocional por encima de lo corporal, esta línea de investigación que se ha abierto resulta prometedora para alcanzar una definición de las emociones acorde con su naturaleza. 
En resumidas cuentas, y esta sería nuestra propuesta después de considerar las posiciones actuales, sus argumentos y contra-argumentos, debe concebirse a las emociones como un fenómeno originario. Su originariedad consiste en que no se dejan reducir ni a meras sensaciones o percepciones, como las teorías puras del sentir proponen, ni a meras evaluaciones, juicios o juicios de valor, como apuestan las teorías cognitivistas radicales, ni a combinaciones de elementos como la teoría de las emociones como como creencias y deseos o la teoría componencial de Ben-zéev. Sensaciones, percepciones, jucios, evaluaciones, creencias y deseos, son todos ellos elementos que juegan un papel en la experiencia emocional, pero no son su última esencia.

Las emociones se caracterizan frente a todos estos otros fenómenos con los cuales están en relación por dos aspectos. Estos dos momentos han sido el hilo conductor a lo largo de este ensayo. Nos referimos, por un lado, al aspecto corporal según el cual las emociones son sentidas con una cualidad singular y específica propia de cada una de ellas. Por otro lado, tenemos el aspecto cognitivo-intencional, según el cual las emociones se fundan en otros actos como juicios, suposiciones, creencias, percepciones, fantasías, recuerdos, etc. y se dirigen a un tipo especial de objetos formales: los valores. La función de las emociones consistiría en orientarnos en el mundo motivando nuestras acciones y fundando ellas mismas actos cognitivos. Ambos aspectos se dan en el fenómeno emocional en unidad y consonancia y sólo son separables a un nivel analítico. Ellos son, por tanto, lo que define e individua a las emociones.

\section{REFERENCIAS BIBLIOGRÁFICAS}

BEN-ZE'EV, A. 2000: The Subtility of Emotions, Massachusetts: MIT Press.

BRENTANO, F. 1924: Psychologie vom empirischen Standpunkt, Tomo I, Leipzig: Felix Meiner.

BRENTANO, F. 1921: Vom Ursprung sittlicher Erkenntnis, Hamburg: Felix Meiner. DESCARTES, R. 2006: Las pasiones del alma, Madrid: Tecnos.

DE SOUSA, R. 1987: The Rationality of Emotion, Cambridge: Cambrige Macs.

DÖRING, S.A. \& MAYER, V. 2002: Die Moralität der Gefühle, Berlin: Deutsche Zeitschrift für Philosophie, Sonderband 4: Akademie Verlag.

DÖRING, S.A. \& PEACOCKE, C. 2002: «Handlungen, Gründe und Emotionen», en Döring, S.A. \& Mayer, V. 2002: Die Moralität der Gefühle, Op. cit., pp. 81-104.

ELSTER, J. 1999: Alchemies of the Mind, New York: Cambridge University Press.

FRANK, M. 2002: Selbstgefühl. Eine historisch-systematische Erkundung, Frankfurt a.M.: Suhrkamp.

GEIGER, M. 1974: «Beiträge zur Phänomenologie des ästhetischen Genusses», Jahrbuch für Philosophie und phänomenologische Forschung I, Tübingen: Max Niemeyer Verlag, Tübingen, pp. 1-118. 
GOLDIE, P. 2002: The Emotions. A Philosophical Exploration, Oxford: Clarendon Press.

GREEN, O. H. 1992: The Emotions, Dordrecht, Boston, London: Kluwer Academic Publishers.

GREENSPAN, P. 1980: "A case of mixed feelings: ambivalence and the logic of emotion», en Rorty, Amélie O. (ed.), Explaining Emotions, Berkeley y Los Angeles: University of California Press, pp. 223-250.

GRIFFITHS, P.E. 1998: What Emotions really are, Chicago: Chicago University Press.

HELM, B. 2002: «Felt Evaluations», en American Philosophical Quarterly, 39, pp. 13-30.

HUSSERL, E. 1992: Logische Untersuchungen, Tomo 2, II. Parte, Obras Completas 4 (Hua. XIX/2), Hamburg: Felix Meiner.

JAMES, W. 1905: The Principles of Psychology, Tomo II, New York: Henry Holt and Company.

JAMES, W. 1967: «What is an emotion?», en Lange, C.G. \& James, W.: The Emotions, New York and London: Hafner Publishing Company.

JOHNSTON, M. 2001: «The Authority of Affect», Philosophy and Phenomenological Research, Vol 63, n. 1, pp. 181-214.

KENNY, A. 1963: Action, Emotion and Will, London: Routledge \& Paul.

KOLNAI, A. 1974: «Der Ekel», Jahrbuch für Philosophie und phänomenologische Forschung X, Tübingen: Max Niemeyer, pp. 119-175.

KÜLPE, O. 1912: Über die moderne Psychologie des Denkens, en Internationale Monatschrift für Wissenschaft, Kunst und Technik. Reimpreso en Külpe, O. (1922), Ed. De Karl Bühler: Vorlesungen zur Psychologie. Leipzig: Hirzel.

LANGE, C.G. 1967: «The Emotions. A Psychophysiological Study», en Lange, C.G. and James, W.: The Emotions, Hafner Publishing Company, New York and London.

LANDWEER, H. 2004: «Phänomenologie und die Grenzen des Kognitivismus», Deutsche Zeitschrift für Philosophie, 52, 2, pp. 467-486.

LYONS, W. 1980: Emotion, Cambridge: Cambridge University Press.

MEINONG 1968: Abhandlung zur Werttheorie, Tomo III, Graz: Akademische Drucku. Verlagsanstalt.

MULLIGAN, K. 1998: «From Appropriate Emotions to Values», The Monist, 81, 1, pp. 161-188.

MULLIGAN, K. 2004: «Husserl on the "Logic" of Valuing, Values and Norms», en Centi, B. \& Gigliotti, G.: Fenomenologia della Ragion Practica. L'Etica di Edmund Husserl, Naples: Bibliopolis, pp. 177-225.

NUSSBAUM, M. 2005: The Upheavals of Thought. The Intelligence of Emotions, Cambridge: Cambridge University Press.

ORTEGA Y GASSET, J. 1966: Obras Completas. Tomo VI. Madrid: Revista de Occicente.

PFÄNDER, A. 1922: Zur Psychologie der Gesinnungen, Halle: Verlag von Max Niemeyer.

PRINZ, J. 2004: Gut Reactions. A perceptual Theory of Emotion. New York: Oxford University Press. 
PUGMIRE, D. 2005: Sound Sentiments: Integrity in the Emotions, Oxford: Oxford UP.

RATCLIFFE M. J. 2005: «The Feeling of Being», Journal of Consciousness Studies, 12 (8-10), pp. 43-60.

SCHMITZ, H. 1981: System der Philosophie:, Tomo 3, 2, Bonn: Bouvier.

SCHMITZ, H. 1998: Der Leib, der Raum und die Gefühle. Stuttgart: Tertium.

SLABY, J. 2004: «Nicht-reduktiver Kognitivismus als Theorie derEmotionen», Handlung, Kultur und Interpretation. Zeitschrift für Sozial- und Kulturwissenschaften, 13, 1, pp. 50-85.

SLABY, J. 2008: Gefühl und Weltbezug. Paderborn: Mentis.

SOLOMON, R.C. 1993: The Passions: Emotions and the Meaning of Life, Indianapolis: Hacket.

STEIN, E. 1917: Zum Problem der Einfühlung, Halle: Buchdruckerei des Waisenhauses.

STEINFATH, H. 2002: «Handlungen, Gründe und Emotionen», en Döring, S.A \& Mayer, V., Die Moralität der Gefühle, op. cit., pp. 105-122.

STOCKER, M. 1987: «Emotional Toughts», en American Philosophical Quarterly, 24 (1), pp. 59-69.

STOCKER, M., con HEGEMAN, E. 1996: Valuing Emotions, New York: Cambridge University Press.

STUMPF, C. 1928: Gefühl und Gefühlsempfindung, Leipzig: Verlag von Johann Ambrosius Barth.

TAPPOLET, C. 2000: Émotions et Valeurs, Paris: Presses Universitaires de France.

TAYLOR, G. 1985: Pride, Shame and Guilt. Emotions of Self-assessment. Oxford: Clarendon Press.

TITCHENER, E. B. 1980: Textbook of Psychology, New York: Scholars' Facsimiles \& Reprints Delmar.

VENDRELL FERRAN, Í. 2008: Die Emotionen. Berlin: Akademie Verlag.

WOLLHEIM, R. 1999: Emotionen. Eine Philosophie der Gefühle, München: C.H.Beck

WUNDT, W. 1920: Grundriss der Psychologie, Stuttgart: Alfred Kröner.

ÍngRID VendRell FerRAn es doctora en Filosofía por la Freie Universität Berlin y está adscrita a la Universidad Complutense de Madrid.

Publicaciones recientes:

Die Emotionen, Akademie Verlag, Berlín, 2008.

«Zwischen Phänomenologie und analytischer Philosophie: Aurel Kolnai», en Accarino, B., \& Schloßberger, M. (Hrgs.): Expressivität und Stil. Helmuth Plessner Sinnes- und Ausdrucksphilosophie, Berlin, 2008.

«Möglichkeiten von Frauen in der ersten Phase wissenschaftlicher Schulenbildung. Emotionen und Sozialität en der frühen Phänomenologie», Feministische Studien, 1/ 2008 
Línea de Investigación:

Antropología filosófica, Fenomenología, Filosofía del S. XIX y XX, Filosofía analítica de las emociones.

\section{Dirección postal:}

DFG-Forschungsstipendiatin, Université de Genève, Swiss Center for Affective Sciences, 7, Rue des Battoirs, 1205 Genève, Suiza.

Dirección electrónica: ingridvefe@web.de 